\title{
Opportunities and Challenges of Information Security Faced by the Era of Big Data
}

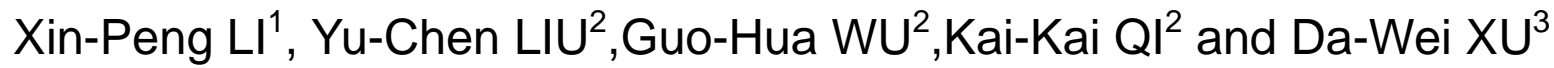 \\ ${ }^{1}$ State grid jibei electric power company \\ ${ }^{2}$ China Electric Power Research Institute \\ ${ }^{3}$ State grid jibei information and telecommunication company
}

KEYWORDS:The era of big data;Information technology security;Challenges and opportunities

\begin{abstract}
With the continuous development of information technology in China, and the status of network information has been promoted, and information data has already infiltrated into different fields such as the economy, education, culture and so on. As an important way to collect and process the use of information, the promotion of information technology with large data is obvious to all. Big data processing technology can solve the set of large capacity data which conventional software can not handle and even can not capture. However, it provides convenience for people's life and communication, but also facing serious challenges of information security. Because of the wide range of people involved and with more information, once the information be revealed, the harm will be difficult to restore. Thus in the era of big data, we have to grasp and solve the core issue of data security facing the challenge and seizing the opportunity, so that this technology can serve more people.
\end{abstract}

\section{INTRODUCTION}

The rapid change of information network technology offering the developing opportunities for our country,and at the same time, it is also changing our work and life quietly. Information technology has now become a kind of important strategic resource which can guarantee us to seize the initiative on the world stage to obtain sufficient voice. As the world's most famous consulting firm, McKinsey has proposed and predicted the arrival of the era of big data as early as a few years ago. And it is visible that data has penetrated into every detail of the field of industry, and even control the production efficiency. In this case, we proposed higher goal with the presence of the massive data in the side which is difficult to master and use, striving to dig deeper and consolidating the security of existing data to build a more solid "data building".

\section{Analysis of big data}

In 2012, "big data"has come into the eyes of people. In early February the New York Times published an article devoted to the analysis of Era of Big Data. The article predicts that big data will be fully used into production and life of human, especially in the field of business. Business decisions and changes in science and technology in the future will have a close relationship with big data.

Usually, big data is not alone but combined with cloud computing. Because the simple big data can not be collected by the people that can not be used for classifying information, and it is not natural to be converted into an important data for people to consult. Such capacity of analysis on big data needs more ability of powerful data calculation and analysis to adapt to play its value. 
According to the real statistics data, it is shown that until the end of 2012, a total of 3.23ZB data generated by the world. The average allocation is about the amount of 400GB data to each person's head. And this huge amount of information is still growing exponentially each year. It is expected that in 2020, the world will produce data which will be at least 40 times now, and the effective information will account for more than $85 \%$.

Big data stands quietly in the forefront of the new century, but also has its unique data performance. Firstly, data types increased rapidly in the era of big data which not only containing the web log, text information, photographs and other traditional information in the resources of network, but also adding a large number of short video, audio, films and TV shows, documentaries, radio and other various media forms of communication. All in all, the traditional media has been fully ported to the platform of information internet. Since then, people's way of life will have a great change with highly convenient network data and massive information transmission to start-up the gold cycle of "T" era of network .

The emergence of electricity suppliers, the succession on the line of network media, the start-up of network drama, network interaction and expansion of the field for resource sharing platform become symbols flowing to the internet with high density and huge data. At the same time, the explosive growth of the data,has higher requirements for the processing speed in new era of data. Data analysis and mining have become urgent needs to overcome the problems of technology. In order to classify, organize and utilize the useful information in the data of low value and density, it is needed to support the structure of the algorithm. Because of the increasing value of the data in the emerging, network information security has gradually entered into the people's sight and gradually received people's attention.

\section{Information security issues faced by the era of big data}

\subsection{Security problem of network information}

As is known to all, generation and collection of huge amount of information in"big data"are all around the Internet and PC expansion. With information technology as the center, using big data in the field of production and consumption, and in the process of change there are many economic and trade with the use of online trading channels. Admittedly, this convenient and efficient way also increases the risk of information leakage. When wealth is transferred from the real life to the virtual Internet, the unpredictable and potential threats are gradually increasing. Massive data is the characteristics and advantages of the era of big data, but it also brings the problems about security of information dissemination.

For example, hackers can steal private information of others through electronic information technology and the Internet channel in addition to the thousands of miles away. And the virtual nature of the information determines that it can't be as effective as the real currency on security defense. Therefore, with the convenient information interaction, it also should see the serious gap of information security and defense behind the truth which is urgent to be made up.

\subsection{Resource allocation problem in massive data}

In the interactive system of mass data and convenient Internet, resource allocation and mobilization have become more frequent and flexible,because it not only combines the modern cloud storage and networking architecture as well as intelligent equipment and networking terminal configuration based on network awareness, identification technology and so on. A large number of high and new technology has been carried out around the big data which is still expanding and derived among them. This makes the global data resources increasingly tense, so that on the analysis of the effectiveness of information transmission and the massive resource allocation where have no shortcoming, the problem has become increasingly prominent. The subversive change of resource 
allocation making the problem of data resources related to the information exchange and operation efficiency and affecting the development of the industry.

\subsection{Storage problem of large capacity private information}

In the era of big data, whether it is government agencies, business units, schools, communities or individuals, data storage is completed by the computer. Whether it is the storage of business transaction information recorded by the enterprises, production information, contract information and even customer information and account information, the problem of storage of the data information storage is need to attach great attention which is similar to the storage of school about teachers and students' information.If financial information and official information stored by Government facing the problem of data leakage,such as flowing into the hands of certain people, it will produce great harm to the enterprises, schools, government and even to the whole community.

\subsection{Illegal transaction of information}

With the high popularity of the Internet in recent years, the base of Internet user has grown to a very terrible number. And such a huge user group is associated with a greater wealth of information data which makes the considerable number of sensitive personal data or business data become the main targets of illegal information transaction. For example, "C-trip Travel" has issues of information leakage appeared in the aspect of security. Detailed travel programs and payment operations, which are registered by the travel platform have been recorded. Then hackers use the defense vulnerabilities of platform data storage entered into the management system successfully, all information of personal data and the redeployment of the user's payment were sold to illegal traders making the property of many users being lost. What's more, personally identifiable information trafficking also continue to emerge in recent years, and many Internet companies facing problem about leakage of registered user's identity information, thereby becoming false account and malicious consumer credit events causing great impact for the victims of personal privacy and life. This kind of economic wealth brought by information transaction creating illegal data business and become the problem of Internet interaction.

\section{Opportunities in the era of big data}

\subsection{Construction of new information security defense system}

Under the background of big data technology, the analysis method on information security is different from the previous information collection and analysis. And from the mass of huge data finishing the classification of information in the process in order to get a parallel information string, then carries on horizontal and vertical analysis of the system which can comprehensively covers to demand information. It can draw the risk of the trend from a large number of data and mathematical calculations for the risk assessment. What's more, according to risk cases and risk categories to develop risk prevention and control programs, the application of large data technology can be completed perfectly. In addition, big data technology can effectively realize the combination of business data and security data, and through reverse encryption measures, it will crumble to bits of data with rearrange made into a large number of invalid information followed by packaged for storage or sent packing. This new type of encryption security protection basing on features of data technology, operators can significantly reduce the risk of information leakage and enhance the level of information security.

\subsection{Anti-hacking technology based on big data}

In the past time, for factors including highly covert and low-key hackers, viruses, and phishing sites, users can do nothing but to face loss of personal privacy and property, and then collect the information, identify the virus source and site IP for clearing these "hidden". However, this passive 
defense and investigate can not really solve the problem, so problem of Network information security still exists.

In fact, the best network information security defense measures in the era of big data lie in the analysis of big data. Based on the data analysis and big data information extraction technology, the system can find the source of data through clues generated by the network attack behavior.So as to avoid the risk before loss happened. At the same time, the transfer of defense system, blockade of IP data source and stop all illegal access can greatly promote the level of safety of the system. In addition, integrated processing technology of big data can sum up high quality of modification program for the protection of network which will upgrade its level for the best data storage and information encryption.

In addition, criminals tend to use large amounts of data and wide range of information to take attacks.Peak data in short time leads to recognition error and access error, which offer an opportunity for the criminals. Similarly, the information security can also be taken to protect large data. For example, it can establish a split access mechanism, which has the ability to link more layer protection to avoid the possibility of a vacuum phase of defense. Large data analysis can be taken as a recognition algorithm framework, in the aspects of user identification and access to the right to obtain a combination of decoding settings, so that a single code stream attacks can not directly obtain the password information.

\section{CONCLUSION}

In the era of big data, challenges and opportunities run parallel. We need to correctly understand the value and significance of information security through the continuous improvement of information security architecture and information interaction network to realize constantly improvement of the relevant security technology which contribute to efficient use of mass information in era of big data, while reducing the occurrence of network security incidents and reducing potential risks in network information security with that a broader space for development can be earned.

\section{REFERENCES}

[1] Wang Jinglei. Discussion on the virtual reality technology in the teaching of computer application [J]. Journal of Taiyuan City Vocational College, 2014,10:118-119.

[2] is a country, hair down. Application of virtual reality technology in computer maintenance in the course teaching of Fujian [J]. computer, 2012,02:193-194.

[3] Rong Jianting. Virtual reality technology in computer assembly and maintenance of teaching reflection on the application of [J]. in secondary occupation education, 2012,10:35-36.

[4] Zhang Ming, Wang Shuying, Guo Tao. The virtual reality technology in the practice of computer application in the teaching of professional talents of [J]. 2011,12:105..

[5] [J]. Journal of Jiangxi Institute of Education Guo Huiru. The application of virtual reality technology in computer teaching experiment, 2013,06:26-29.

[6] Chen Zhiyu. Virtual reality technology in experiment teaching application [J]. Journal of Gansu Lianhe University (NATURAL SCIENCE EDITION), 2013,02:103-106.

[7] Yanzhen. VR virtual reality technology in computer teaching applications [J]. Journal of Jincheng Institute of Technology, 2011,02:21-23.

[8] Liu Xuyong. Application of virtual reality technology in experimental teaching [J]. value engineering, 2011,35:150-151. 